\title{
Calcified chronic subdural hematoma: illustrative case
}

\author{
Rupesh Pakrasi, MBBS, ${ }^{1}$ Payoz Pandey, MCh, ${ }^{2}$ Srijan Das, MBBS, ${ }^{3}$ Shreya Datta, ${ }^{4}$ and Dipti Saha ${ }^{5}$ \\ Departments of ${ }^{1}$ General Surgery and ${ }^{2}$ Neurosurgery, Calcutta National Medical College and Hospital, West Bengal, India; ${ }^{3}$ Medical College and Hospital Kolkata, West Bengal, \\ India; ${ }^{4}$ Calcutta National Medical College and Hospital, West Bengal, India; and ${ }^{5}$ Nirratan Sircar Medical College and Hospital, West Bengal, India
}

\begin{abstract}
BACKGROUND Calcified chronic subdural hematomas (CCSDHs) are rare variants of chronic subdural hematomas (CSDHs) accounting to only $0.3-2.7 \%$ of CSDHs. Although the majority of the patients with CSDHs recover from surgery, there still is some doubt about its being applied to CCSDHs.

OBSERVATIONS In this case report, the authors present a case of a 75-year-old male presenting with deterioration of motor function in his left limbs over the course of 18 months and acute neurological deterioration in the form of altered sensorium for 7 days. The patient experienced an episode of aspiration in the preoperative period that led to deterioration of pulmonary function in the postoperative period. A chest radiograph showed diffuse patches suggesting pulmonary compromise. Computed tomography and magnetic resonance imaging (MRI) documented a large subdural collection at the right frontal and parietal hemisphere with calcification, which was successfully and completely removed by surgery.

LESSONS The chances of a subdural hematoma progressing to calcification is extremely rare. The presentation of this case was such that surgical intervention was the only option left for the patient. The presence of lacunar infarcts in the thalamus on MRI can also be attributed to the calcified hematoma.
\end{abstract}

https://thejns.org/doi/abs/10.3171/CASE21468

KEYWORDS calcified chronic subdural hematoma; surgery; CT; MRI

Chronic subdural hematoma (CSDH) is one of the most common neurosurgical conditions, but calcified chronic subdural hematoma (CCSDH) is a rare variant of the same. ${ }^{1,2} \mathrm{CCSDH}$ was first described by a Bohemian pathologist in $1884 .^{3}$ The clinical presentation also varies, with some patients remaining asymptomatic and others having seizures, dementia, and other complications, especially in bilateral calcified subdural hematomas, also referred as armored brain. ${ }^{4,5}$ The mechanism of calcification of the periphery of some of the subdural hematomas is likely to be multifactorial, involving both vascular and metabolic factors. ${ }^{6}$ The calcification in CSDHs is caused mainly by overdrainage of cerebrospinal fluid, which still remains very rare. ${ }^{7}$ Surgical treatment is the best treatment for CSDHs; however, for $\mathrm{CCSDHs}$, the neurosurgical excision of the hematoma can be problematic due to the adherence of the calcific hematoma to the leptomeninges and underlying cortical surface in armored brain. The removal of the calcification in such cases may damage the underlying cortex and disturb the whole neurological homeostasis. ${ }^{8-11}$ Literature reviews suggest that surgery cannot improve long-standing symptoms. Hence, surgery is recommended only when acute or progressive neurological symptoms occur. $^{12,13}$

Here, we report the surgical outcome of a 75-year-old male with deterioration of motor function in his left limbs over the course of 18 months and acute neurological deterioration in the form of altered sensorium for 7 days with an episode of aspiration in the preoperative period. A large subdural collection at the right hemisphere with calcified inner membrane was successfully and completely removed by surgery, which was followed, however, by pulmonary complications due to an episode of aspiration in the preoperative period.

\section{Illustrative Case}

We report a case of a 75-year-old male presenting with deterioration of motor function in his left limbs over the course of 18 months and acute neurological deterioration in the form of altered sensorium for 7 days. He was transferred to our department from the medicine ward of Calcutta National Medical College with a Glasgow Coma Scale (GCS) score of E1V2M5, left hemiparesis.

ABBREVIATIONS CCSDH = calcified chronic subdural hematoma; $\mathrm{CSDH}=$ chronic subdural hematoma; $\mathrm{CT}=$ computed tomography; GCS = Glasgow Coma Scale; $M R I=$ magnetic resonance imaging. 


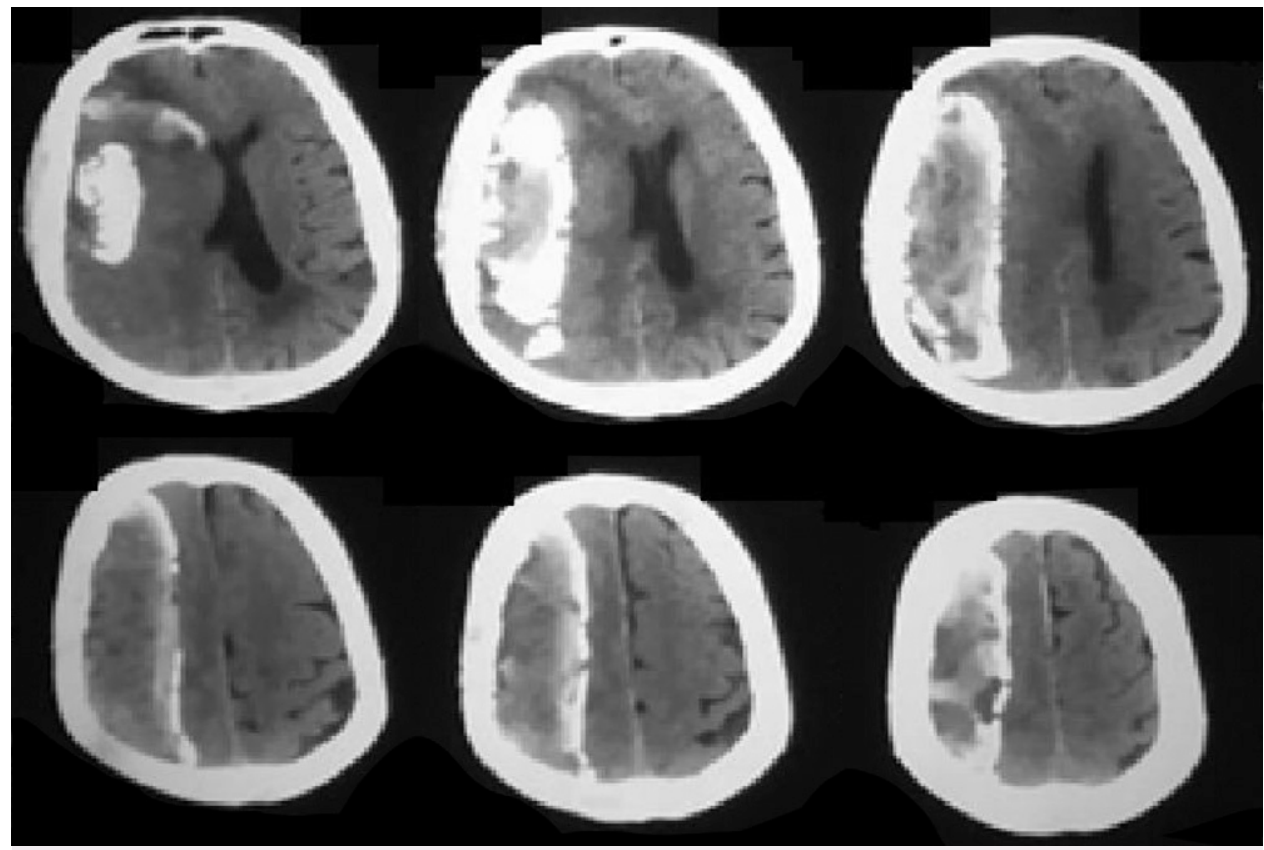

FIG. 1. Preoperative computed tomography scans of the patient.

Computed tomography (CT) and magnetic resonance imaging (MRI) documented a large subdural collection of the right hemisphere with inner calcification (Fig. 1).

An MRI scan showed an organized, right-sided frontal and parietal hematoma with calcification and mass effect (Fig. 2). Lacunar infarcts were present in both thalami along with definite focal ischemic changes in the frontal white matter. A right front parietal craniotomy was performed (Fig. 3), exposing an "armored dura" with the mold of the underlying hematoma.

The dura was opened in a curvilinear manner, exposing the calcified capsule of the CSDH, which was tightly adherent to the inner surface of the dura. After it was dissected, the capsule was incised, exposing various stages of subacute hematoma. The calcified hematoma was removed en bloc (Fig. 4).

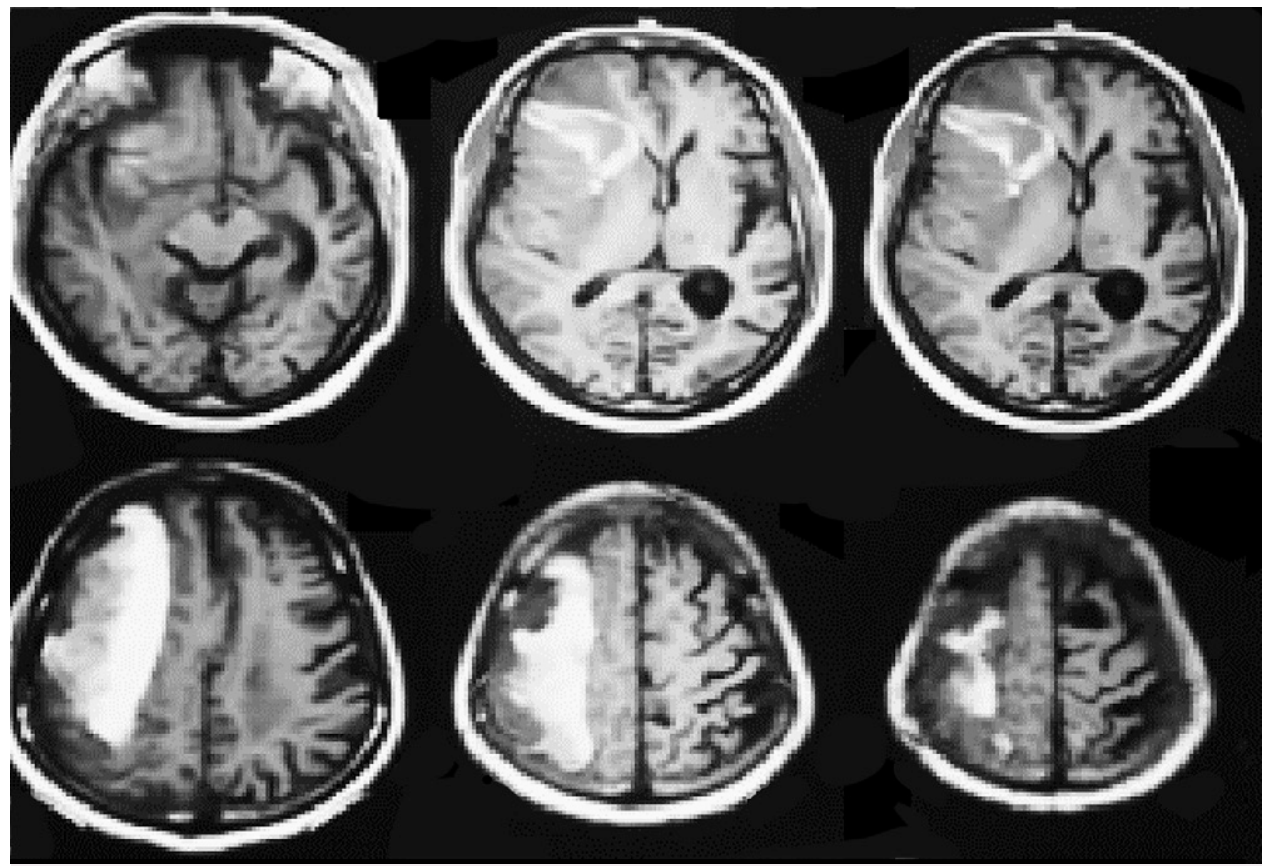

FIG. 2. Preoperative MRI scans of the patient. 


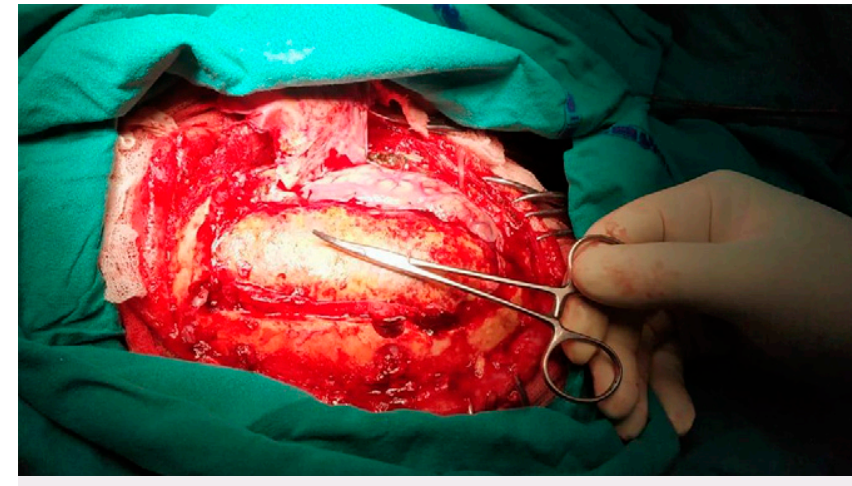

FIG. 3. Right frontal craniotomy was performed with hemostatic forceps pointing to the $\mathrm{CSDH}$.

However, the arachnoid membrane was intact and not adhered to the hematoma, allowing its complete removal without injuring the underlying brain. In the postoperative period, the pulmonary function of the patient deteriorated further, eventually leading to respiratory distress followed by an episode of pneumonia. The pulmonary complications led to the death of the patient on postoperative day 4 as a result of respiratory arrest.

\section{Discussion}

\section{Observations}

CSDH is defined as the accumulation of blood between the dura mater and arachnoid mater of the brain and is often associated with insignificant injury that presents with symptoms 2 weeks after the inciting incident. ${ }^{14}$ The initial presenting symptoms are often unnoticed because there is vague onset of cognitive impairments, headaches, and somnolence along with focal neurological deficits on the

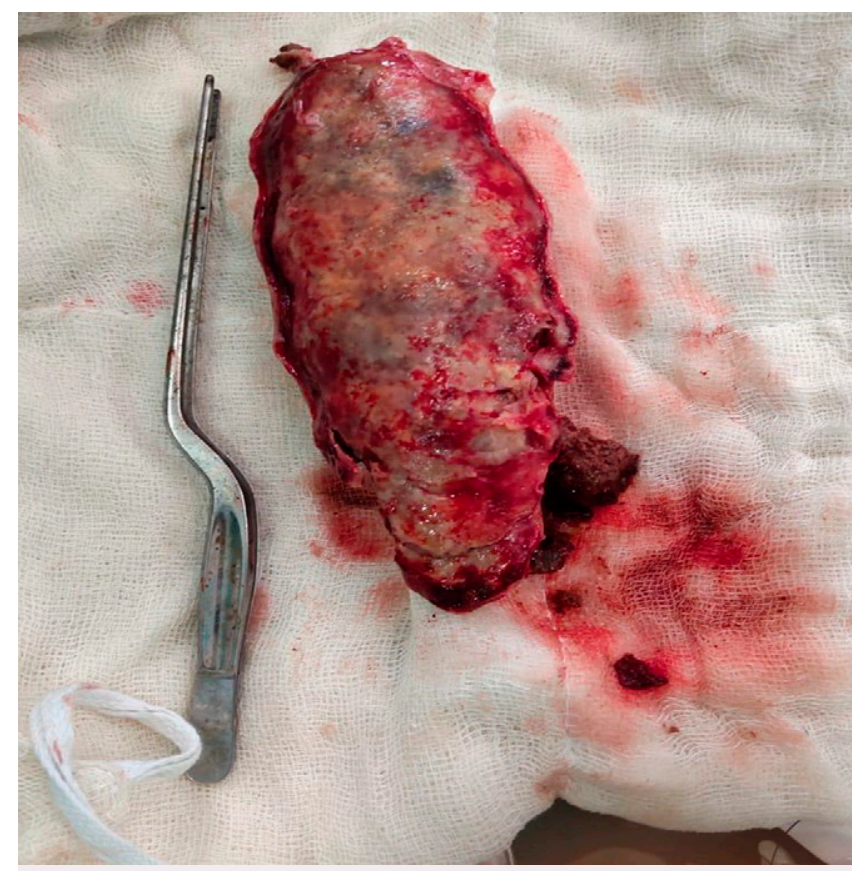

FIG. 4. Extracted specimen of subdural hematoma. contralateral side of the lesion. The exact pathophysiological mechanism behind the development of CSDH is unknown, but the greater incidence among the elderly population is attributed to agerelated brain atrophy, ${ }^{15}$ increased risk of fall-related injuries, ${ }^{16}$ and the frequent use of blood-thinning agents. ${ }^{17}$ In our reported case, there was also reported lacunar infarcts bilaterally in the thalamus along with focal ischemic changes in the left frontal white matter. The exact cause of association of CSDH with lacunar infarcts is not fully elucidated, but it has been concluded in one study that distortion, twisting, and/or elongation of the lenticulostriate arteries by the $\mathrm{CSDH}$ in the elderly, especially in patients with a variety of comorbidities, such as diabetes mellitus and hypertension, are one of the major risk factors for these infarcts. ${ }^{18}$

Prior studies conducted on CSDH showed that the connective tissue membrane surrounding a subdural hematoma is hyalinized, or calcified hematomas have been found. ${ }^{19}$ Therefore, this association of CSDH with total calcification makes this case very novel for better understanding of the pathophysiology, the treatment intervention, and the expected outcome of $\mathrm{CCSDH}$.

The etiology of a CCSDH is as yet unknown, but a variety of studies suggest a multifactorial effect of poor circulation and absorption in subdural space along with intravascular thrombosis, which plays a role in calcification and ossification. ${ }^{20}$ Studies have reported that poor circulation under the subdural hematoma content leads to deposition of calcium particles and hyalinization of the connective tissue. These microscopic calcium deposits pile up, leading to eventual serious calcification, which occurs approximately 6 months after the initial hemorrhage, albeit influenced by a variety of individual factors. ${ }^{21}$ Ossification is the terminal stage of the calcification process and is rarely reported as such.

CSDHs are characterized by slow progression of a variety of neurological symptoms and associated sensorineural deficits. ${ }^{22}$ Similar incidences of calcified subdural hematoma in the past revealed complaints of dementia, seizure, mental retardation, growth retardation, persistent headache, and left hemiparesis with hyperreflexia on neurological examination. Papilledema and/or pathological reflexes may also be noted, ${ }^{6}$ but sometimes incidentally patients may be asymptomatic. $^{6}$ In our case, the patient had experienced left-sided hemiparesis for the past 18 months and acute neurological loss in the form of altered sensorium for the past 7 days.

CT and MRI invariably reveal large subdural collections at the right frontal and parietal hemispheres with calcification.

Because the inner layer was thicker and compressing the brain seriously, the use of only fluid drainage would not have been able to improve the symptoms or help with reexpansion of the brain. Therefore, at this stage, craniotomy and excision of the calcified hematoma en bloc were the only viable options. In most of the cases, removal of the hematoma may damage the brain, depending on the adhesion between the hematoma capsule and the brain. Also, a few studies have recommended excluding elderly patients from any surgical intervention due to occurrence of symptoms as a result of cerebral atrophy ${ }^{11}$ combined with the common postoperative complications of recurring hemorrhage based on insufficient brain expansion after prolonged compression. ${ }^{8}$ Hence, surgical intervention for managing CCSDH is still considered controversial. ${ }^{10}$ In our case, we were able to avoid any substantive brain injury because the arachnoid membrane was unadhered to the hematoma; therefore, reexpansion of the brain was possible. In the preoperative period, the patient had an episode of aspiration. Chest radiographic findings hinted at pulmonary 
compromise leading to postoperative complications that culminated in respiratory arrest and ultimately the death of the patient.

\section{Lessons}

A plausible conclusion that can be derived from this case is that though calcification of the subdural hematoma is a very rare occurrence, its surgical treatment and successful removal are possible, depending on the adhesion between the hematoma capsule and the brain visualized by MRI. Surgery may be the first-line intervention for treating CCSDHs. The patient in this case had aspiration, which increased his susceptibility to the development of postoperative pneumonia. The stress of general anesthesia was a probable additive factor that led to the ultimate respiratory compromise in spite of the adequate treatment modalities that were offered.

\section{References}

1. Yadav YR, Parihar V, Namdev H, Bajaj J. Chronic subdural hematoma. Asian J Neurosurg. 2016;11(4):330-342.

2. Xiao Z, Chen X, Li K, Zhang Z. Calcified chronic subdural hematoma: a case report and literature review. Transl Neurosc Clin. 2017;3(4):220-223.

3. Von Rokitansky C. Handbuch der Pathologischen Anatomie. Braumüller \& Seidel; 1846.

4. Dammers R, ter Laak-Poort MP, Maas Al. Neurological picture. Armoured brain: case report of a symptomatic calcified chronic subdural haematoma. J Neurol Neurosurg Psychiatry. 2007;78(5): 542-543.

5. Rahman $A$, Haque $M$, Bhandari PB. Calcified chronic subdural haematoma. BMJ Case Rep. 2012;2012:bcr0120125499.

6. Pappamikail L, Rato R, Novais G, Bernardo E. Chronic calcified subdural hematoma: case report and review of the literature. Surg Neurol Int. 2013;4:21.

7. Tuli S, Drake J, Lawless J, Wigg M, Lamberti-Pasculli M. Risk factors for repeated cerebrospinal shunt failures in pediatric patients with hydrocephalus. J Neurosurg. 2000;92(1):31-38.

8. Sgaramella E, Sotgiu S, Miragliotta G, Fotios Kalfas Crotti FM. "Matrioska head": case report of calcified chronic subdural hematoma. J Neurosurg Sci. 2002;46(1):28-31.

9. Pruna V, Bucur N, Neacsu A, et al. Calcified chronic subdural hematoma: case report. Rom Neurosurg. 2008;15(2):22-25.

10. Ito M, Saito S, Kondo R, Nagahata M, Kayama T. A case of calcified chronic subdural hematoma wherein MRI was useful for decision of the treatment strategy. Article in Japanese. No Shinkei Geka. 2014:42(8):717-722.

11. Kaplan M, Akgün B, Seçer HI. Ossified chronic subdural hematoma with armored brain. Turk Neurosurg. 2008;18(4):420-424.

12. Oda S, Shimoda M, Hoshikawa K, Shiramizu H, Matsumae M. Organized chronic subdural haematoma with a thick calcified inner membrane successfully treated by surgery: a case report. Tokai J Exp Clin Med. 2010;35(3):85-88.

13. Yan HJ, Lin KE, Lee ST, Tzaan WC. Calcified chronic subdural hematoma: case report. Changgeng Yi Xue Za Zhi. 1998;21(4): 521-525.

14. Karn M, Yonghang S. Acute on chronic bilateral subdural hematoma in a woman with a remote history of previous subdural hematoma managed by trephination: a case report. JNMA J Nepal Med Assoc. 2020;58(228):607-610.

15. Liu H, Yang Y, Xia Y, et al. Aging of cerebral white matter. Ageing Res Rev. 2017;34:64-76.

16. Lin JT, Lane JM. Falls in the elderly population. Phys Med Rehabil Clin N Am. 2005;16(1):109-128.

17. Connolly BJ, Pearce LA, Hart RG. Vitamin K antagonists and risk of subdural hematoma: meta-analysis of randomized clinical trials. Stroke. 2014;45(6):1672-1678.

18. Yamada SM, Tomita Y, Takaya Y. Lacunar infarction caused by chronic subdural hematoma. Neurol Med Chir (Tokyo). 2020;60(8): 397-401.

19. Anegawa S, Torigoe R, Furukawa Y, Harada K. Chronic calcified subdural hematoma - report of a case. Article in Japanese. No Shinkei Geka. 1987;15(11):1249-1254.

20. Norman $\mathrm{CH}$ Jr, Dubowy J. Chronic calcified subdural hematoma. J Natl Med Assoc. 1971;63(1):3-5.

21. Afra D. Ossification of subdural hematoma. Report of two cases. J Neurosurg. 1961;18(3):393-397.

22. Gelabert-González M, Iglesias-Pais M, García-Allut A, MartínezRumbo R. Chronic subdural haematoma: surgical treatment and outcome in 1000 cases. Clin Neurol Neurosurg. 2005;107(3): 223-229.

\section{Disclosures}

The authors report no conflict of interest concerning the materials or methods used in this study or the findings specified in this paper.

\section{Author Contributions}

Conception and design: Pakrasi, Pandey. Acquisition of data: Pandey. Analysis and interpretation of data: Pandey. Drafting the article: Pakrasi, Pandey, Datta, Saha. Critically revising the article: Pandey, Das, Datta, Saha. Reviewed submitted version of manuscript: all authors. Approved the final version of the manuscript on behalf of all authors: Pakrasi. Statistical analysis: Pandey. Administrative/technical/ material support: Pandey. Study supervision: Pakrasi, Pandey, Das.

\section{Correspondence}

Rupesh Pakrasi: Calcutta National Medical College and Hospital, West Bengal, India. amsepindia@gmail.com. 